\title{
Utilisation de la géomatique pour l'analyse des risques liés à la dynamique du trait de côte à Kribi
}

\author{
Romaric Emmanuel OUABO ${ }^{1}$, Roberteau Serges TCHOFFO $^{2}$, Bryan Ralain NGATCHA ${ }^{3}$ \\ ${ }^{1}$ Life and Earth Sciences Institute, Environmental Management, Pan African University, University of \\ Ibadan, Nigeria. \\ ${ }^{2}$ Department of GIS and spatial Sciences, UICN (Union International pour la Conservation de la Nature), \\ Yaoundé, Cameroon. \\ ${ }^{3}$ Life and Earth Sciences Institute, Mineral Exploration, Pan African University, University of Ibadan, \\ Nigeria
}

\section{RESUME}

Kribi est une ville côtière de 80957 habitants qui est caractérisé par une activité économique en pleine expansion. Elle fait face à une dynamique du trait de côte entrainant des impacts. Le présent travail a pour objectif l'analyse des risques liés à cette dynamique côtière par la géomatique.

Une revue de la littérature aboutira à une évaluation du milieu physique. La seconde phase était la descente de terrain ou un ensemble d'interviews a été conduit avec des cibles (mission d'étude pour l'aménagement de l'océan, Communauté Urbaine et les leaders traditionnels). La localisation des endroits à risque a été faite à l'aide d'un GPS et analysé avec les images satellites. Les résultats obtenus montrent que les côtes rocheuses, sableuses et sablo-rocheuses sont dominantes. Les risques liés à la dynamique du trait de côtes sont les risques naturels et ceux exacerbés par la dynamique côtière (effondrements, détérioration des écosystèmes). Cette détérioration des écosystèmes s’appuie sur des aléas naturels, le bilan érosifs des matériaux et la pression humaine importante qui se traduit par le non-respect de la règlementation. En vue de préserver cette zone et limité l'impact de l'érosion côtière, il est urgent de mettre en œuvre un plan de gestion intégré.

Mots clés : Image satellitaire, Dynamique côtière, Trait de côte, Risque, Kribi.

Received: 10/05/2018

Accepted: $11 / 06 / 2018$

DOI: https://dx.doi.org/10.4314/jcas.v14i2.3

(C) The Authors. This work is licensed under the Creative Commons Attribution 4.0 International Licence. 


\begin{abstract}
Kribi is a coastal town of 80,957 inhabitants which is characterized by a booming economic activity. It faces a dynamic coastline resulting in adverse impacts. The present work aims to analyse the risks related to these coastal dynamics by geomatics.

A review of the literature lead to an assessment of the physical environment. The second phase was the field work where interviews were conducted with targets (study mission for the development of the ocean, Urban Community and traditional leaders). The location of the areas at risk were done using a GPS and data analysed with the satellite images. The results obtained show that the rocky, sandy and sandy-rocky coasts are dominant. The risks associated with coastline dynamics are natural hazards and those exacerbated by coastal dynamics (collapse, deterioration of ecosystems). This deterioration of ecosystems is based on natural hazards, the erosive balance of materials and the significant human pressure that results in non-compliance with regulations. In order to preserve this area and limit the impact of coastal erosion, it is urgent to implement an integrated management plan.
\end{abstract}

Key words: Satellite image, Coastal dynamics, Coastline, Risk, Kribi. 


\section{INTRODUCTION}

La gestion des zones côtières a suscité au cours des dix dernières années, un intérêt considérable et obtenu une place de choix dans les débats internationaux relatifs à la conférence des nations unies sur l'environnement et le développement (CNUED) de 1992 tenue à Rio De Janeiro, au Sommet de Johannesburg sur le Développement Durable de 2002 et à la mise en œuvre du chapitre 17 de l'Agenda 21. La gestion durable des écosystèmes côtiers constitue à différentes échelles un enjeu primordial. Dans tous les pays du monde, la côte exerce un attrait sur les hommes et leurs activités (Tchawa, 2004, Paskoff, 2010). Important foyer de peuplement, la côte est aussi une zone de transfert ou d'ouverture sur l'extérieur. Elle suscite convoitise, fait l'objet de compétition pour l'accès et le contrôle de ses multiples richesses. La difficulté à maitriser les processus naturels à l'œuvre dans cette zone s'explique par le fait qu'il s'agisse d'un milieu d'interface par excellence. En effet, la côte est une zone où interagissent des processus de nature et d'origine très diverses : atmosphériques : (pluies, vents); hydrodynamiques: (houle, marrées, courants); morpho lithologiques : (profil et hauteur de la côte, nature des roches); biosphériques: (type et densité de la végétation littorale) anthroposphériques: (formes d'occupation et type de mise en valeur). La gestion côtière est la complexe interaction les lois, les programmes et les efforts d'évaluation des écarts et de prise de décision sur le mode d'exploitation, de préservation et d'évaluation des ressources et des opportunités de la zone côtière (Sikirou, 1997 ; Nfomou et al, 2004 ; Tiafack et al, 2014). Le développement durable des zones côtières repose sur la gestion efficace des risques, à l'heure actuelle et dans le contexte des impacts croissants du changement climatique. La nécessité d'améliorer notre compréhension de l'aléa et du risque, en mettant à disposition des outils pour mieux renseigner les politiques d'aménagement et d'urbanisation et de ce fait améliorer la gestion du risque, est essentielle.

La ville de Kribi regorge d'un grand nombre d'enjeux et on remarque de plus en plus une certaine remontée des eaux vers le continent (Tchawa, 2004). Certaines infrastructures se situent dans la zone rouge interdite de construction par les pouvoirs publics. Dès lors quels outils peuvent être mis à la disposition des acteurs afin de palier à ce défi croissant avec le temps?

Le but de ce travail est d'apporter aux décideurs des outils fiables et adaptés à ce milieu à risque qu'est Kribi pour sa gestion intégrée durable. Il est important de mentionner que notre étude se limitera aux risques liés à la dynamique du trait de côte et les impacts y relatifs.

\section{II- MILIEU PHYSIQUE}

1) le milieu physique de Kribi

La ville côtière de Kribi est située au bord du golfe de Guinée, dans la Région du Sud au Cameroun et dans le Département de l'Océan dont elle est le chef-lieu, à l'embouchure de la rivière Kienké. Les coordonnées de Kribi sont: $2^{\circ} 56^{\prime} 6.00^{\prime \prime} \mathrm{N}, 9^{\circ}$ 54' 36.00"E (Latitude:2.9350; Longitude:9.9100). Elle est à environ $150 \mathrm{~km}$ au sud de Douala. Kribi borde le vaste plateau Sud-Camerounais. Elle est bâtie sur des plateaux côtiers de faibles altitudes constitués essentiellement de roches sédimentaires que l'on retrouve dans la plaine côtière. On relève la présence des côtes rocheuses, des cotes sableuses, des cotes vaseuses, sablo-rocheuses et cambrés. Les reliefs les plus bas se situent à l'Ouest de la région au contact de l'océan. C'est une bande de terrain sédimentaire haute de moins de 100 mètres. Elle continue vers l'Est à un niveau compris entre 100 à 300 mètres d'altitude (Tiafack, 2014 ; Mena et al, 2016).

La présente étude concerne la partie de la côte du Cameroun qui va de Campo à l'embouchure du

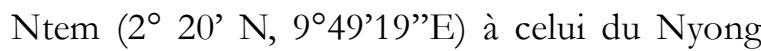
$\left(3^{\circ} 15 ’ 36^{\prime} " \mathrm{~N}: 9^{\circ} 53^{\prime} 46^{\prime \prime} \mathrm{E}\right)$. Vers l'intérieur du 
pays, les limites à considérer sont celles des communes d'arrondissement de Campo, de Kribi rurale, et de Kribi urbaine (Fig1).

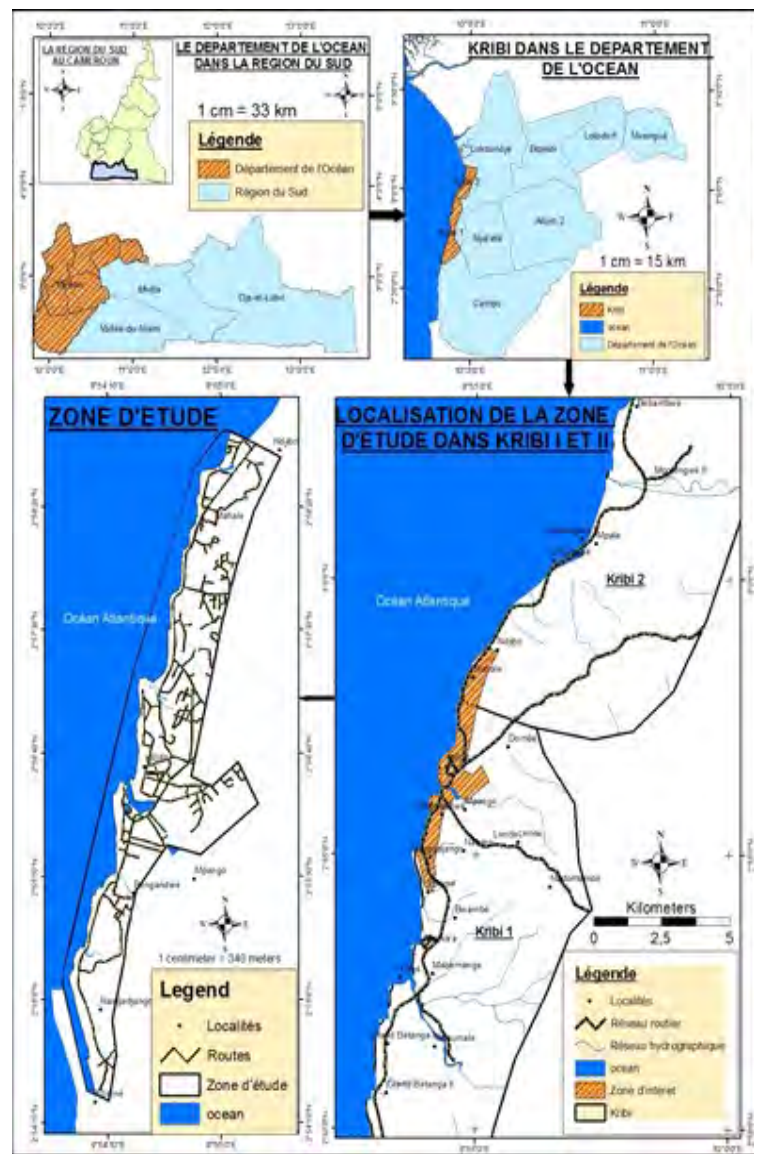

Fig. 1 : Zone d'étude du projet.

Source : Quick bird image/INC

\section{- Le climat}

A Kribi règne un climat équatorial de type guinéen caractérisé par l'existence de quatre saisons (Mena et al, 2016), soient 02 saisons sèche (de décembre à mars pour la grande saison et de juillet à août pour la petite saison) et 02 saisons de pluies (de septembre à novembre pour la grande saison et de avril à juin pour la petite saison). La durée moyenne quotidienne de l'insolation varie de 8 à 10 heures toute l'année.

Les moyennes températures maxima quotidiennes oscillent entre $25^{\circ} \mathrm{C}$ et $33^{\circ} \mathrm{C}$ tandis que les moyennes des températures minima quotidiennes se situent entre $15^{\circ} \mathrm{C}$ et $22^{\circ} \mathrm{C}$ (Suchel, 1998 ; Mena, 2008 ; Mena et al, 2016).

\section{- Les précipitations}

Le régime des précipitations a des tendances induites par les quantités de précipitations au fil des années (Mena et al, 2016).

- Les années à tendances peu pluvieuses (1979 1993) avec des moyennes des pluies de 2628,26 $\mathrm{mm}$ (inférieur à la moyenne qui est de $2862,84 \mathrm{~mm})$.

- Les années à tendance moyennement pluvieuse (1960-1978) avec des moyennes de 2845,24 mm. Elle est proche de la moyenne.

\section{- Les températures}

La moyenne des températures est de $26,83^{\circ}$

Celsius avec un maximum de $28,16^{\circ}$ Celsius en 1998 et un minimum de $24,85^{\circ}$ Celsius en 2006 (Tiafack et al, 2014).

\section{- La végétation}

Du Sud vers le Nord on distingue :

La forêt biafréenne sempervirente à caesalpiniaceae. Les forets littorales de basse altitudes se trouvent à la même latitude, mais plus rapprochée de la cote. Ce premier grand ensemble est ourlé par les mangroves de l'embouchure du Ntem. Plus au Nord, la forêt change peu et reste verdoyante; devenue plus rocheuse, la côte n'est plus apte à porter la mangrove (Tchawa, 2004).

\section{- Géologie et sols}

Le bassin côtier camerounais affleure en trois unités distinctes, à savoir le bassin de Kribi/ Campo, le bassin de Douala et Rio del Rey ou le bassin de Bamusso. Ces bassins font partie de la marge Ouest-Africaine formée lors de l'élargissement progressif du Sud Atlantique par la déchirure continentale Sud-Nord (Ngaha et al., 1987). La côte atlantique se trouve à l'ouest de Kribi, où l'avancement des roches du socle continental (Fig. 2) sépare le trait de côte plus ou moins rectiligne en deux bassins sédimentaires majeurs, les bassins Campo et Douala. Le bassin de Campo au sud constitue la limite nord du bassin Gabo-Equato Guinéen. Il a environ $400 \mathrm{~m}$ de roches sédimentaires déposées pendant la transgression Albienne à Turonienne (environ 
103-90 Ma) sur le socle (Fig 2). Ces roches sont constituées de grès conglomératiques en alternance avec des maërls micacés noirs et bandés et des calcaires oolithiques (Gazel et al., 1956). La roche dominante du socle est le gneiss. Les sols sont généralement profonds, composés de matériaux fortement altérés avec des surfaces sableuses devenant argileuses au fur et à mesure qu'on descend en profondeur. Les sols sont physiquement stables et bien structurés, visiblement bien drainés et très perméables dans l'ensemble.

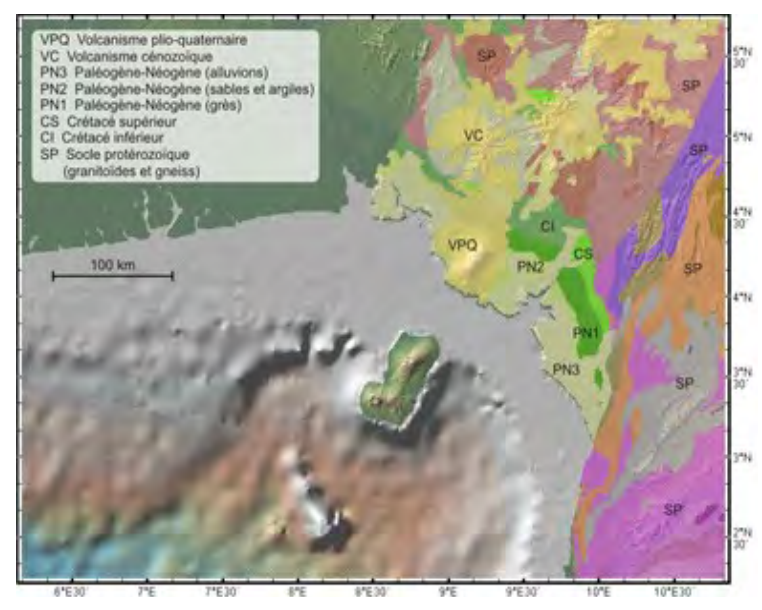

Fig 2. Géologie simplifiée de la côte camerounaise. Source : Cartothèque Lycée Dominique Savio.

\section{- Hydrologie et hydrogéologie}

Elle est dense et constituée de plusieurs cours d'eau (le Nyong, la Lokoundje, la Kienke, la Lobe, le Ntem). Sur la ceinture côtière, les sols sont en partie sableux et très perméables. Le bas niveau des terres et la perméabilité des sols, le réseau hydrographique et la forte pluviométrie dans la zone font conclure à l'existence de nappes phréatiques peu profondes.

\section{2) Méthodologie d'analyse de l'érosion côtière}

Notre méthodologie s'organise autour de trois grandes étapes : Celles-ci vont de la collecte des données bibliographiques à l'analyse et synthèse des résultats en passant par le travail de terrain. 2.1- Première étape : Collecte documentaire Cette phase consiste à se documenter sur toute la littérature traitant des thématiques autour de
Kribi. Ainsi, les bibliothèques et les instituts de recherche telles que l'Institut National de la Cartographie, la bibliothèque du département de Géographie de l'Université de Yaoundé I, la documentation du département de l'environnement, la cartothèque de Dominique Savio, la cartothèque de l'Institut de la Recherche Géologique et Minière, le centre de documentation du MINEPDED ont servi de point de collecte de tous les documents en rapport avec la ville de Kribi et les zones côtières en général. Les documents recherchés se référaient principalement à l'aménagement de ladite zone, l'étude des risques et l'utilisation de la télédétection pour l'étude d'une ligne de rivage. Après avoir balisé le terrain et été éclairé sur cette problématique, une phase de descente sur le terrain a été planifiée. Cette descente a été faite avec quelques petits supports cartographiques (carte administrative de Kribi et spatio-carte pour orientation et la facilitation du travail sur le terrain.

\section{2- Deuxième étape : Descente sur le terrain}

Toucher du doigt la réalité du terrain nécessite une étude empirique. C'est l'occasion de rencontrer les acteurs institutionnels et des collectivités décentralisées en relation avec la problématique des côtes. Ainsi, une rencontre a été possible avec les acteurs suivants :

\section{- La Communauté Urbaine de Kribi à travers} les responsables des services techniques qui au quotidien font face à des problèmes liés aux permis de bâtir et à la protection de l'environnement côtier contre les exploitants de sable ;

- la MEAO (Mission d'Etude et d'Aménagement de l'Océan) qui a présenté brièvement ses missions et les outils mises à sa disposition pour bien les remplir;

- Les délégués départementaux del'environnement et du cadastre ;

- Les pêcheurs et sableurs sur la côte.

L'analyse et la synthèse de toutes les données collectées ont suivi cette descente sur le terrain. 


\section{3- Troisième étape : Le traitement des données et la synthèse des résultats.}

Il a été préalablement question de scanner et saisir toutes les données collectées dont seules les plus pertinentes ont été retenues. à cette phase a donné lieu à l'inventaire et à l'analyse spatiale, réalisées à l'aide des logiciels Erdas Imagine pour les traitements d'image et ArcGIS pour analyses et élaboration des cartes. Ces cartes commentées et l'analyse des résultats obtenus effectuée, le document fin prêt peut être déposé auprès des acteurs de la ville de Kribi.

\section{III- RESULTATS}

\section{1) les types de côtes à Kribi}

Les côtes kribiennes sont caractérisées par trois domaines distincts. Le domaine à vasière, le domaine sableux et le domaine rocheux.

Au niveau de l'hôtel Palm Beach, nous avons une cote à anse, de pente très faible comprise entre $7^{\circ}$ et $9^{\circ}$, délimitée au Sud par des bourrelets rocheux. Au Nord de cette zone, le profil morphologique de la plage devient de plus en plus cambré au point de devenir concave en face de l'hôtel «LE PHARE » où la pente dépasse $15^{\circ}$. Du Sud au Nord sur le plan longitudinal, nous avons les côtes rocheuses, les cotes sableuses de type anse et de rivage cambré. Les vasières sont localisées à hauteur des embouchures.

- Les côtes rocheuses

La côte kribienne fait partie de la ceinture rocheuse du littoral camerounais qui s'étend de Campo à Kribi, au Sud du bassin côtier et de Limbé à Banoussouan. En marée basse, on aperçoit de véritables masses rocheuses à plus de trente mètres de la laisse de basse marée.

- Les côtes sableuses

Elles côtoient les côtes rocheuses ou tout écran protecteur en aval. Leurs formes arrondies font d'elles des baies parfaites.

- Les côtes cambrées

- Les côtes à vasière

- Les côtes sablo-rocheuses
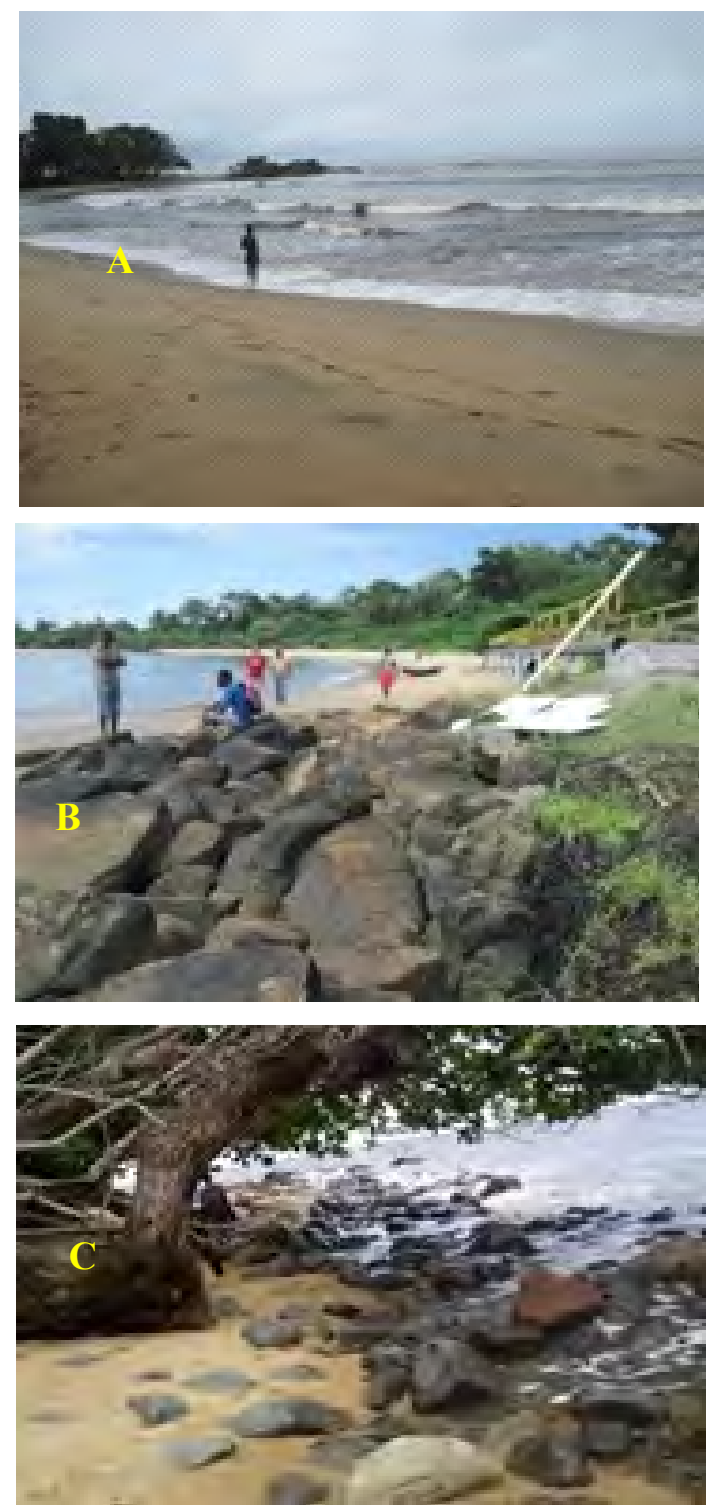

Fig 3 : Photos A, B, C : Quelques types de côtes identifiées à Kribi.

La figure 3 illustre trois types de côte répertoriées dans la zone d'étude. Le type A qui est le type le plus observé constitue les côtes de type sableuses. La figure $\mathrm{B}$ présente les côtes rocheuses vers l'embouchure de la Lobé et la figure $\mathrm{C}$ fait état des côtes sablo-rocheuses.

\section{2) les types de risques liés à la dynamique du trait de côte.}

Les facteurs humains donnent lieu à un certain nombre de risques qui viennent se greffer aux risques naturels. Dès lors les zones vulnérables ont été préalablement modélisées. 


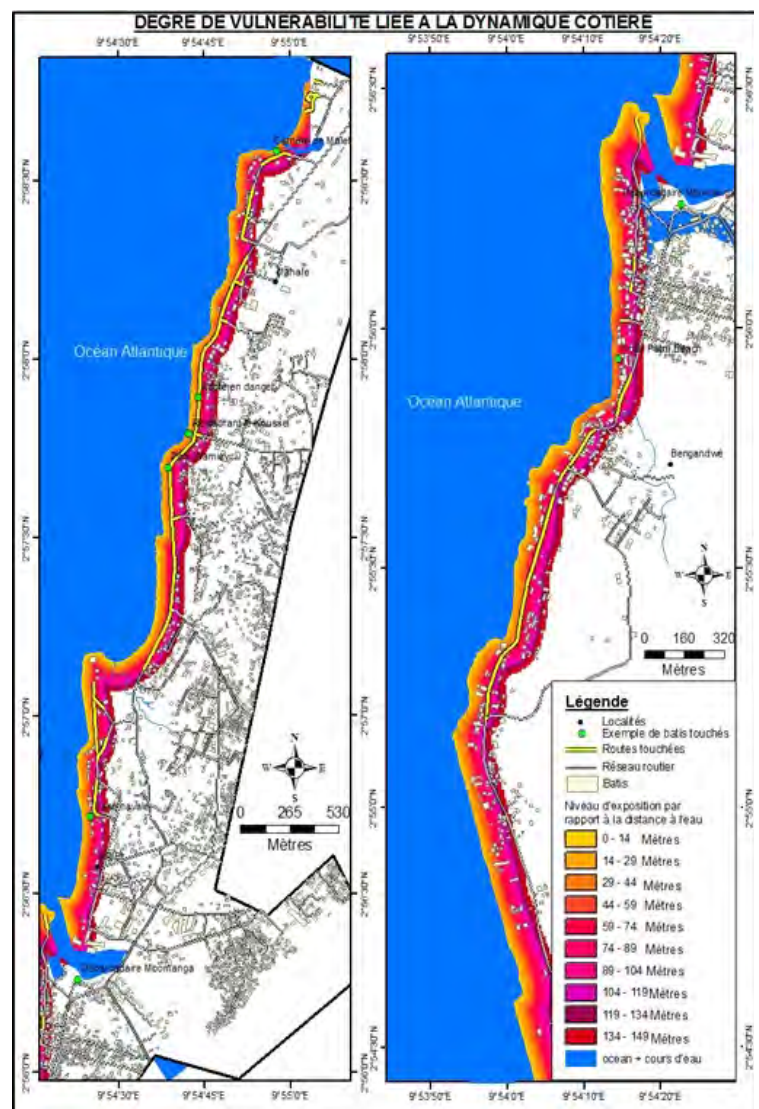

Fig.4 : Les zones vulnérables à la dynamique côtière.

Source : Quick Bird/Auteurs

La figure 4 précise le degré de vulnérabilité de chaque espace en fonction de sa distance par rapport au trait de côte. Dépendamment de la distance au trait de côte, la vulnérabilité réduit passant de 14 à 149 mètres au fur et à mesure qu'on s'éloigne de la côte. Cette progression permet d'échelonner à l'aide des indicateurs l'état de vulnérabilité des structures humaines de ladite zone.

\section{3) les risques naturels en présence}

Certains risques sont déjà observés sur la côte de Kribi.il s'agit principalement du risque de noyade et du risque de chute.

\section{1) Le risque de noyade}

Les plages sont le lieu par excellence pour la baignade des touristes et populations riveraines amateurs de nage. Le constat fait est que certaines personnes ont perdu leur vie de suite de noyade, le risque étant plus élevé en marée haute, période pendant laquelle la mer démontre la dangerosité en envahissant le continent (Cf. photo 1,Fig. 2 ?).

\section{2) Le risque de chute}

Les côtes rocheuses à Kribi constituent un point d'attraction pour les visiteurs qui s'y hissent pour avoir une meilleure vue et garder des souvenirs de leur voyage (cf fig.5), mais non sans risque. En effet ces rochers sont en permanence immergés, ce qui augmente le risque le chute.

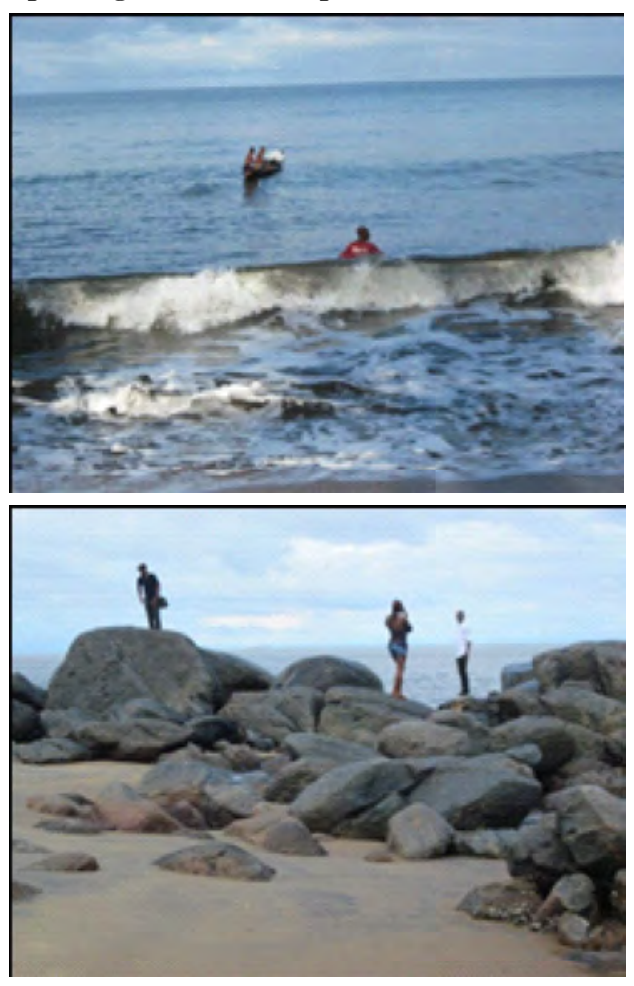

Fig.5 : Photo 01,02 ; Les zones à risque de chute et de noyade. Source : Archive des auteurs 


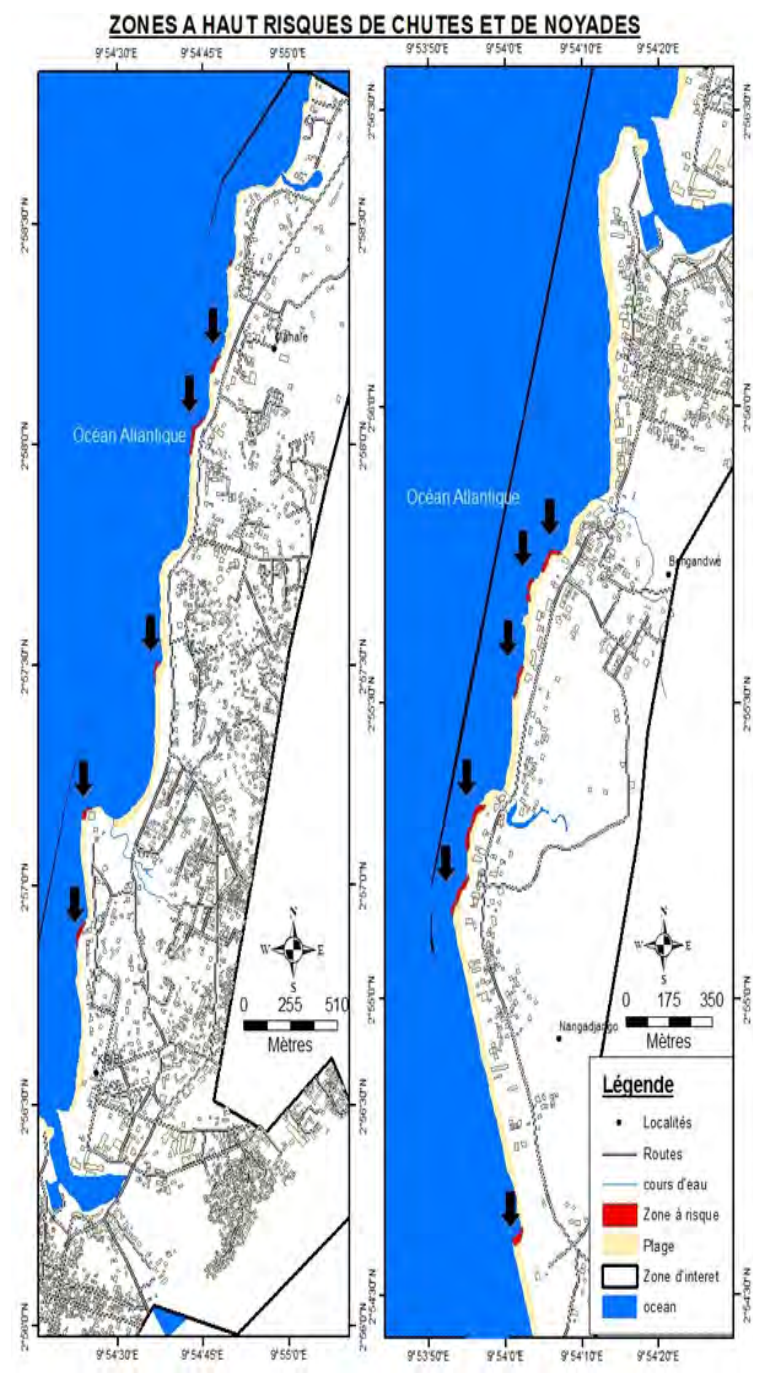

Fig.6 : Zones à risque de chutes et de noyade

La figure 6 fait référence aux zones potentiellement dangereuses pour les visiteurs. Force est de constater que ces zones se trouvent sur des appointements de la ligne littorale car ces zones résistent mieux à l'érosion des vagues. Ces roches constituent un réel bouclier pour le trait de côte.

\section{4) Les risques exacerbés par la dynamique du trait de côte}

La plupart des risques observés est le fruit de la dynamique du trait de côte dont les facteurs ont été présentés.

\section{1) Les risques d'effondrement des infrastructures}

Le long du rivage, des constructions en matériaux définitifs ou non sont observées. Ces dernières subissent des dégradations et des fissures considérables liées à la dynamique côtière et finissent par s'effondrer. La figure 7 ci-après en présente quelques cas.
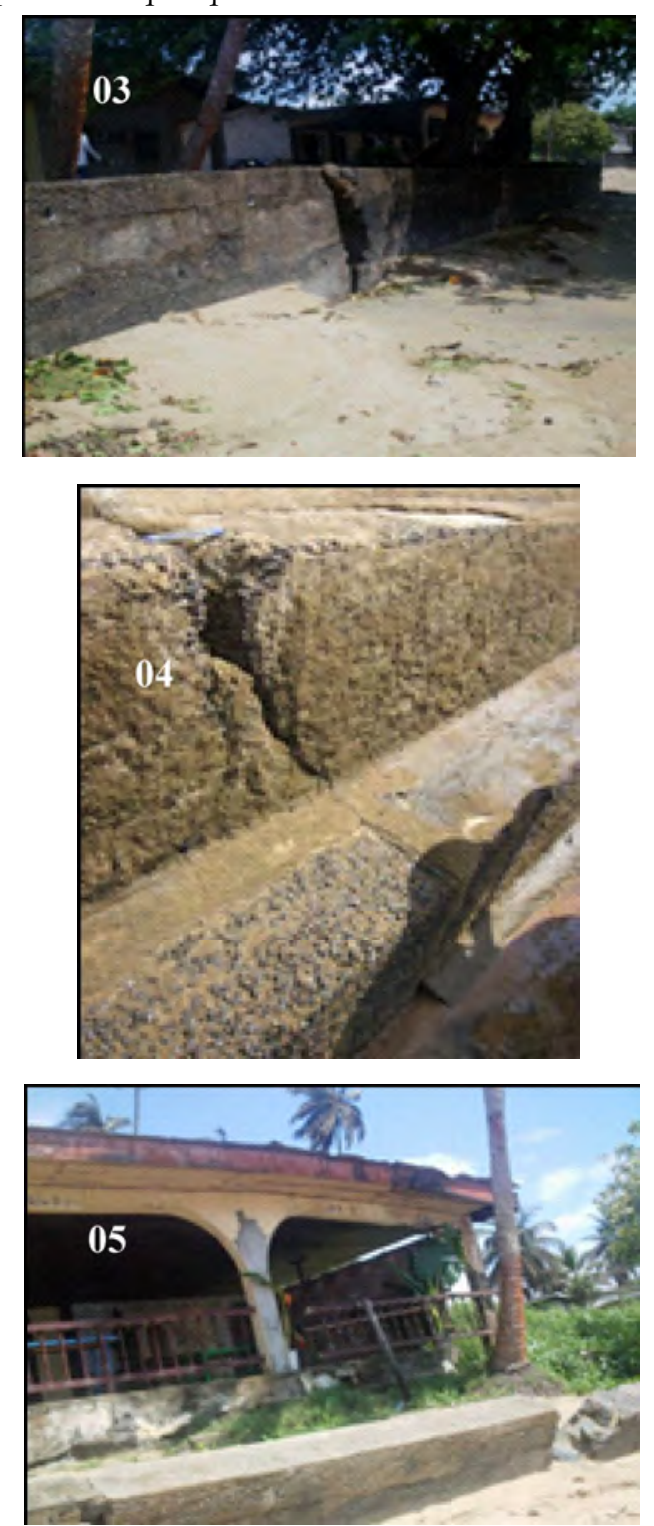

Fig.7 : Photo 03, 04, 05: Le risque d'effondrement des infrastructures.

Les dégâts sont très souvent importants les bâtiments situés en bordure de mer, la plupart étant des établissements hôteliers. Il est également à noter qu'au rythme auquel évolue le trait de côte, il est probable que l'axe principal traversant le Lycée bilingue de Kribi soit rompu.

Du point de vue géologique, les failles sont des zones faibles dans la terre qui sont représentées par certaines caractéristiques géologiques telles 
que le réseau de flux de drainage, les lignes et les contacts lithologiques. Le linéaire structurel est défini comme des caractéristiques géomorphiques linéaires en raison de zones de faiblesse ou de déplacement structurel de la croûte terrestre (Caran et al., 1982). L'identification des failles est un aspect important dans la gestion de l'environnement urbain. Les systèmes de faille actifs peuvent avoir des influences significatives dans les déplacements lents du sol lors de mouvements verticaux ou horizontaux néoorthogonaux ou soudains, à la suite de tremblements de terre de grande magnitude (Ioane et al., 2014). Dans les activités d'urbanisme, en plus d'une zonation sismique détaillée, la connaissance de la répartition géographique des failles actives, et en particulier des failles traversant une ville dans des zones fortement construites comme Kribi, peut être précieuse dans de nombreux cas. Par conséquent, il est nécessaire d'étudier la structure géologique afin d'identifier les systèmes de failles. Selon Caran et al. (1982), les alignements perçus dans les images par télédétection sont des indicateurs fiables de la structure géologique. L'analyse des linéaments/ fractures structurelles donne un aperçu de la caractéristique tectonique locale, mais aussi des informations sur la possibilité d'une évolution tectonique à échelle régionale dans une zone qui n’a jamais été observée. La télédétection intégrée dans une plate-forme SIG (Système d'Information Géographique) a été utilisée pour fournir un aperçu synoptique des caractéristiques géologiques structurelles (linéaments), possiblement reliés ou faille sur la zone de Kribi (Fig. 8). Environ 20 linéaments ont été identifiées sur la zone d'étude (Fig. 8), Mais la majorité d'entre elles ont été nettoyées pour une représentation fluide. Ils ont indiqué des faiblesses dans le sous-sol. Lorsque de grandes failles se rompent et produisent des tremblements de terre, elles déforment généralement la surface du sol. C'est peut-être l'une des causes des fissures sur les murs (Fig.7) dans la zone de Kribi noter au cours de cette étude. La région de Kribi a été témoin d'un certain nombre de tremblements de terre au cours des années. Le dernier a été ressenti le 23 juillet 2002, avec une intensité maximale de $\mathrm{IV}^{+} \mathrm{MM}$ et une magnitude de 3,6 $\pm 0,4$, avec son épicentre situé dans l'océan Atlantique, à 50-55 km à l'ouest de Kribi (Ntepe et al., 2004). L'évaluation préliminaire du risque sismique montre que la ville de Kribi est la zone la plus risquée de la région (Ntepe et al., 2004). Cette information, entre autres, a été utilisée pour délimiter les zones sensibles aux dangers dans la zone d'étude.

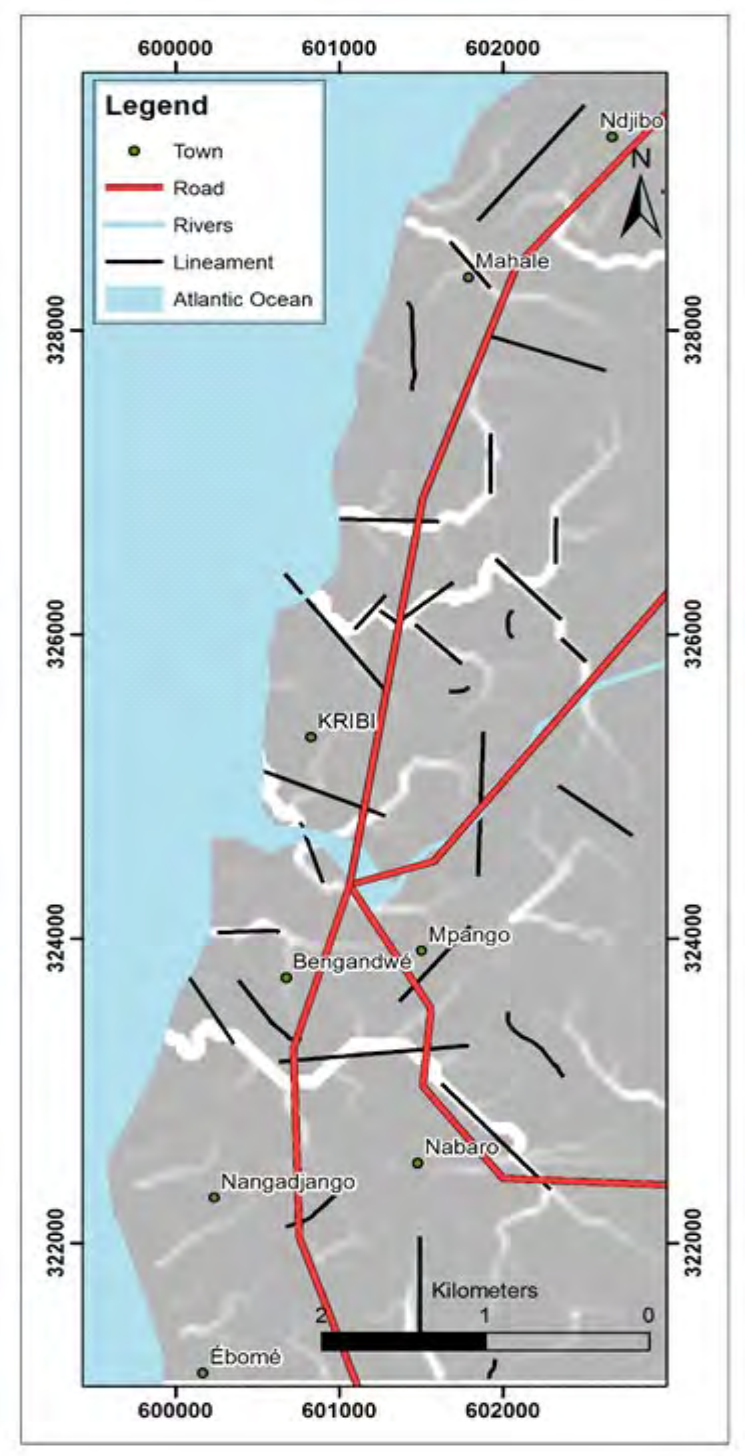

Fig.8: Interprétation des linéaments à partir d'une image ASTER DEM d'une résolution de 30 mètres. 


\section{2) Le risque de détérioration des écosystèmes}

On observe de façon de plus en plus récurrente des écroulements d'arbres sur les plages de Kribi en raison du mouvement du trait de côte. Ces derniers constituent pourtant de bons points d'ombrage pour les touristes. Ils sont d'autant plus importants qu'ils sont en général des arbres fruitiers (cocotiers) dont les fruits très prisés sont commercialisés dans la ville et ailleurs. La chute de ces arbres constitue donc un enjeu environnemental majeur (Cf. Fig.8).
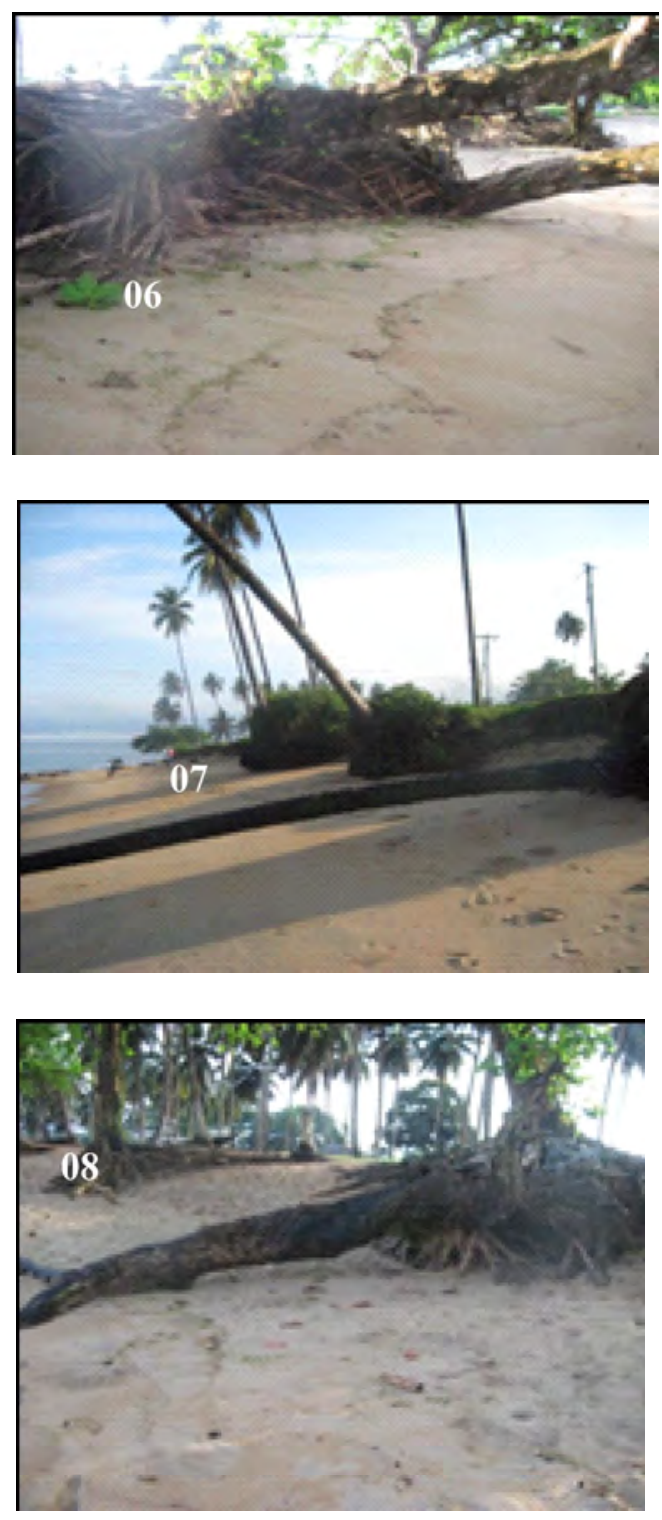

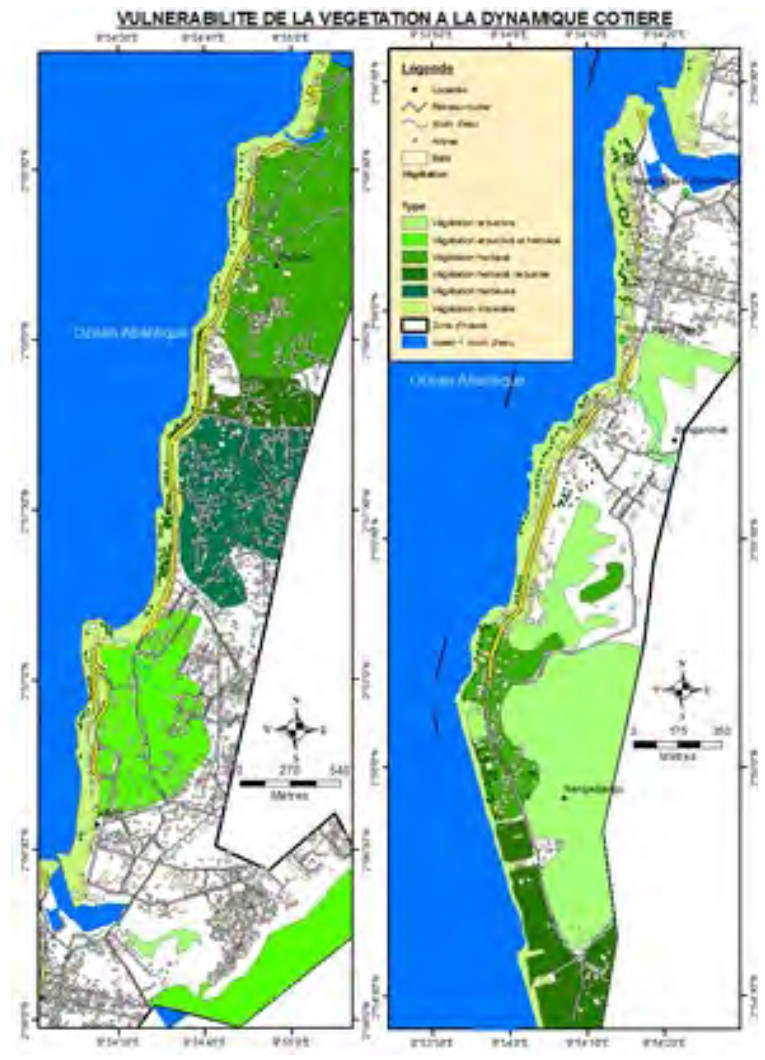

Fig. 10 : Les zones des écosystèmes vulnérables à la dynamique côtière.

La figure 10 présente les types de végétation et les zones de vulnérabilité. Ces écosystèmes jouent un rôle fondamental pour la régulation des microclimats et ainsi, tous les acteurs inclus dans la problématique de la gestion des zones côtières doivent s'impliquer davantage dans leur protection.

Au vu de ce qui précède, la dynamique globale constatée dans la zone de Kribi s'est faite d'une façon progressive, ce qui implique le paramètre temps dans les études pour une dynamique variée. 
Fig.9 : Photo 06, 07, 08 : Chute d'arbres sur une plage de Kribi.

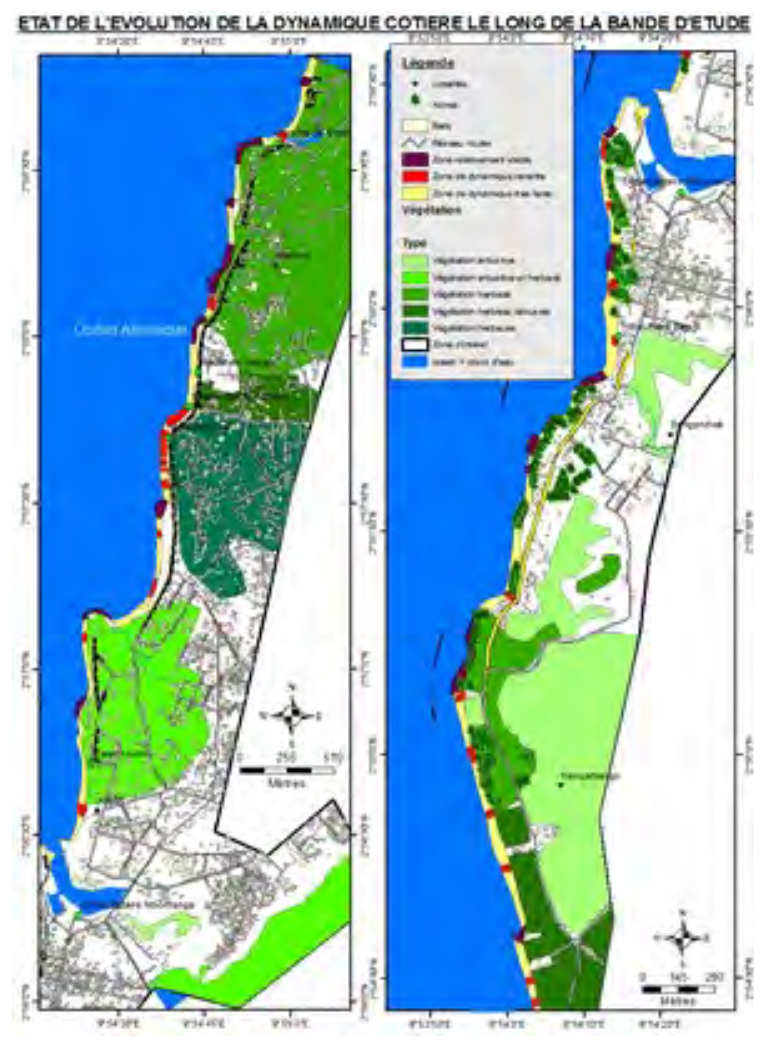

Fig.11 : Evolution globale de la dynamique le long de la bande côtière de Kribi.

La figure 11 permet de classifier la bande côtière en zones stables, zones de dynamique récentes et zones de dynamique lente. Ces différentes zones présentent une dynamique particulière en fonction des catégories.

\section{DISCUSSION}

Globalement, l'élément à risque identifié dans le cadre de cette étude reste la population. Il est question de montrer les manifestations de ce risque. Des chercheurs (Morin \& Kuete, 1989) ont montré un certain nombre de segments rectilignes décalés en échelons par de petits secteurs orthogonaux.

\section{1) Les aléas naturels comme facteur de risque}

\section{a) Le climat}

Le trait de côte de Kribi aujourd'hui en mouvement constitue un réel risque pour les populations. Le premier aléa est naturel avec une ambiance aujourd'hui rythmée par le réchauffement climatique. Kribi est une zone de climat équatorial humide. Le tableau No. 1 cidessous présente les données pluviométriques de Kribi.

Table1 : Tableau climatique de Kribi.

\section{Janvier Février Mars Avril Mai Juin Juillet Août Septembre Octobre Novembre}

\begin{tabular}{|c|c|c|c|c|c|c|c|c|c|c|}
\hline Température moyenne $\left({ }^{\circ} \mathrm{C}\right)$ & 26.9 & 26.5 & 26.526 .5 & 25.9 & 25.4 & 24.5 & 24.5 & 25 & 24.9 & 26 \\
\hline Température minimale moyenn & $\mathrm{e}\left({ }^{\circ} \mathrm{C}\right)$ & 23.7 & 22.722 .7 & 22.7 & 22.6 & 22.6 & 21.8 & 21.8 & 22.7 & 21.8 \\
\hline 22.8 & & & & & & 22.7 & & & & \\
\hline Température maximale $\left({ }^{\circ} \mathrm{C}\right)$ & 30.2 & 30.3 & 30.330 .3 & 29.3 & 28.3 & 27.2 & 27.2 & 27.3 & 28.1 & 29.2 \\
\hline \multicolumn{11}{|c|}{29.2} \\
\hline Température moyenne $\left({ }^{\circ} \mathrm{F}\right)$ & 80.4 & 79.7 & 79.779 .7 & 78.6 & 77.7 & 76.1 & 76.1 & 77.0 & 76.8 & 78.8 \\
\hline \multicolumn{11}{|c|}{78.6} \\
\hline Température minimale moyenn & $\mathrm{e}\left({ }^{\circ} \mathrm{F}\right)$ & 74.7 & 72.972 .9 & 72.9 & 72.7 & 72.7 & 71.2 & 71.2 & 72.9 & 71.2 \\
\hline 73.0 & & & & & & 72.9 & & & & \\
\hline Température maximale $\left({ }^{\circ} \mathrm{F}\right)$ & 86.4 & 86.5 & 86.586 .5 & 84.7 & 82.9 & 81.0 & 81.0 & 81.1 & 82.6 & 84.6 \\
\hline \multicolumn{11}{|c|}{84.6} \\
\hline Précipitations (mm) & 94 & 183 & 238309 & 258 & 130 & 209 & 486 & 482 & 197 & \\
\hline
\end{tabular}

Source: Climate-Model by Climate-Data.Org 
Une différence de $419 \mathrm{~mm}$ est enregistrée entre le mois le plus sec et le mois le plus humide. 2.4 ${ }^{\circ} \mathrm{C}$ de variation sont affichés sur l'ensemble de l'année. Janvier est le mois le plus chaud de l'année avec une température moyenne de $26.9^{\circ} \mathrm{C}$. Le mois le plus froid de l'année est celui de Juillet avec une température moyenne de $24.5^{\circ} \mathrm{C}$.

De même, d'après la même source La station de Kribi enregistre une température moyenne de l'air de $25^{\circ} \mathrm{C}$. Ces moyennes grimpent à $27.5^{\circ} \mathrm{C}$ en saison sèche avec un maximum de $31.5^{\circ} \mathrm{C}$ en mars. La moyenne des températures moyennes minimales se situe autour de $23^{\circ} 4$ contre $32^{\circ} 5 \mathrm{C}$ pour la moyenne des maxima mensuels.

Les enquêtes de terrain confirment une avancée de la mer qui se traduit par la modification du trait de côte.
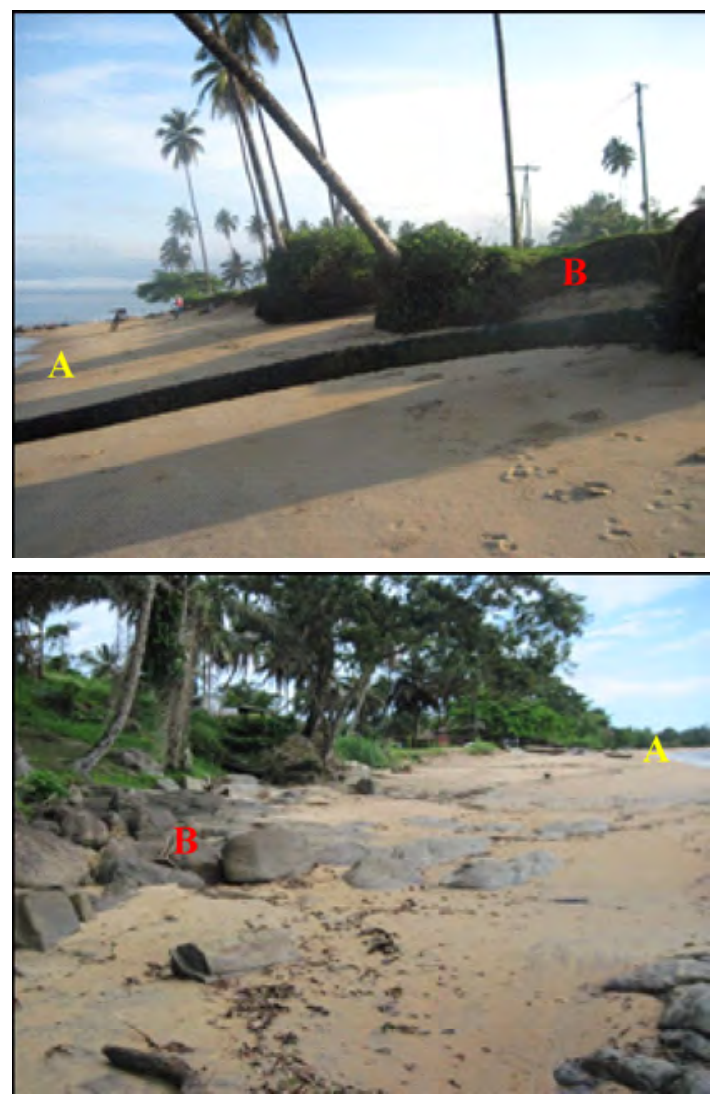

Fig.12 : Photo 09 et 10 : Le trait de côte sous l'influence de la montée de la mer.

Les deux images de la figure 12 présentent la considérable distance entre le point A (Niveau moyen de la mer) et le point $\mathrm{B}$ (trait de côte). Ce qui permet de conclure à une bonne activité liée à la montée de la mer, assurément induites par les changements climatiques à Kribi.

\section{a) Le bilan érosif des matériaux sous l'action de la mer}

La mer est toujours chargée de matériaux solides venant du continent parce que lorsqu'elle frappe sur la côte, elle grignote progressivement ses matériaux constitutifs. Le constat fait est que la ligne de côte est façonnée par le poids, la forme ainsi que la quantité de matériaux qui y déferlent.

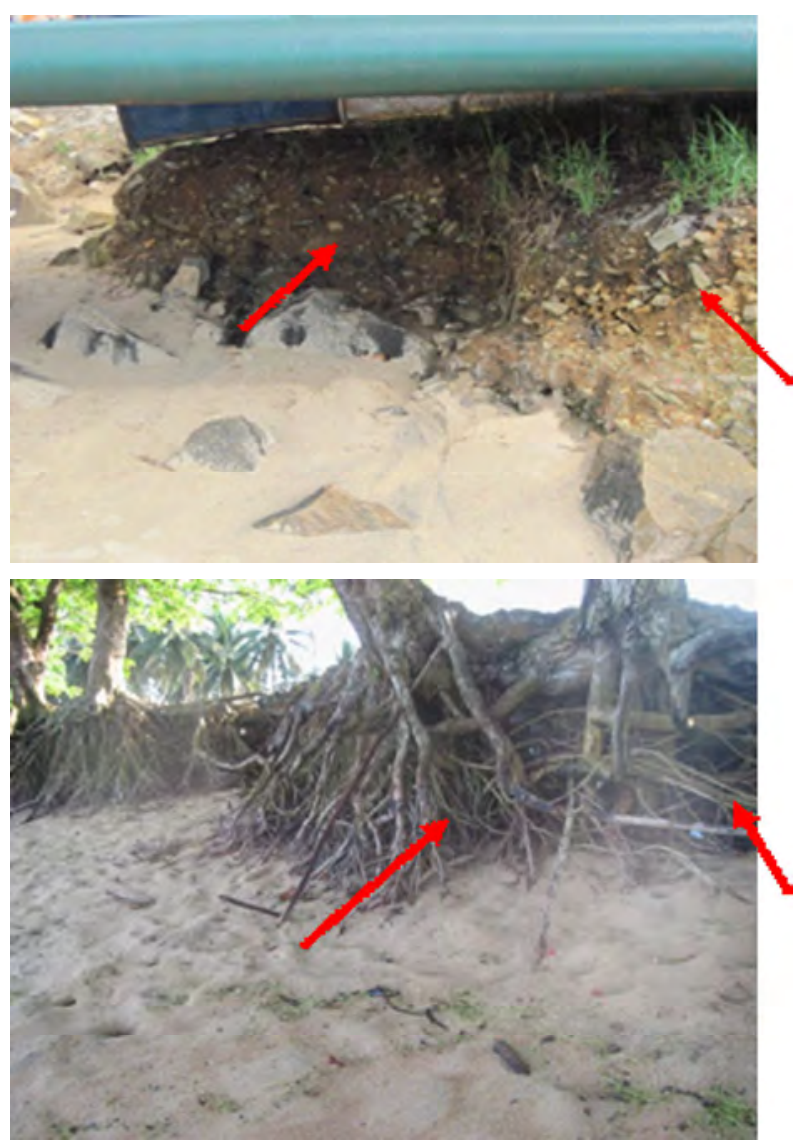

Fig.13 : Photos 11,12: L'activité érosive sur le modelage du trait de côte.

Les flèches rouges de la figure 13 indiquent la direction des vagues et l'effet de l'érosion sur la modélisation du trait de côte et le déchaussement des arbres et des infrastructures.

1) Le facteur humain dans la dynamique du trait de côte

a) L'activité d'extraction de sable L'extraction du sable est une activité malheureusement importante des côtes de Kribi. Les populations locales exploitent le sable à 
longueur de journée sur les plages malgré les récriminations de la communauté urbaine qui les oriente vers le sable des fleuves et rivières. Il est aussi à relever d'après les témoignages que derrière cette activité se cachent beaucoup de gros bonnets qui usent de leur influence pour exploiter le sable, rendant ardue la tâche des agents de la communauté urbaine de Kribi. Cette activité favorise considérablement la dynamique du trait de côte.
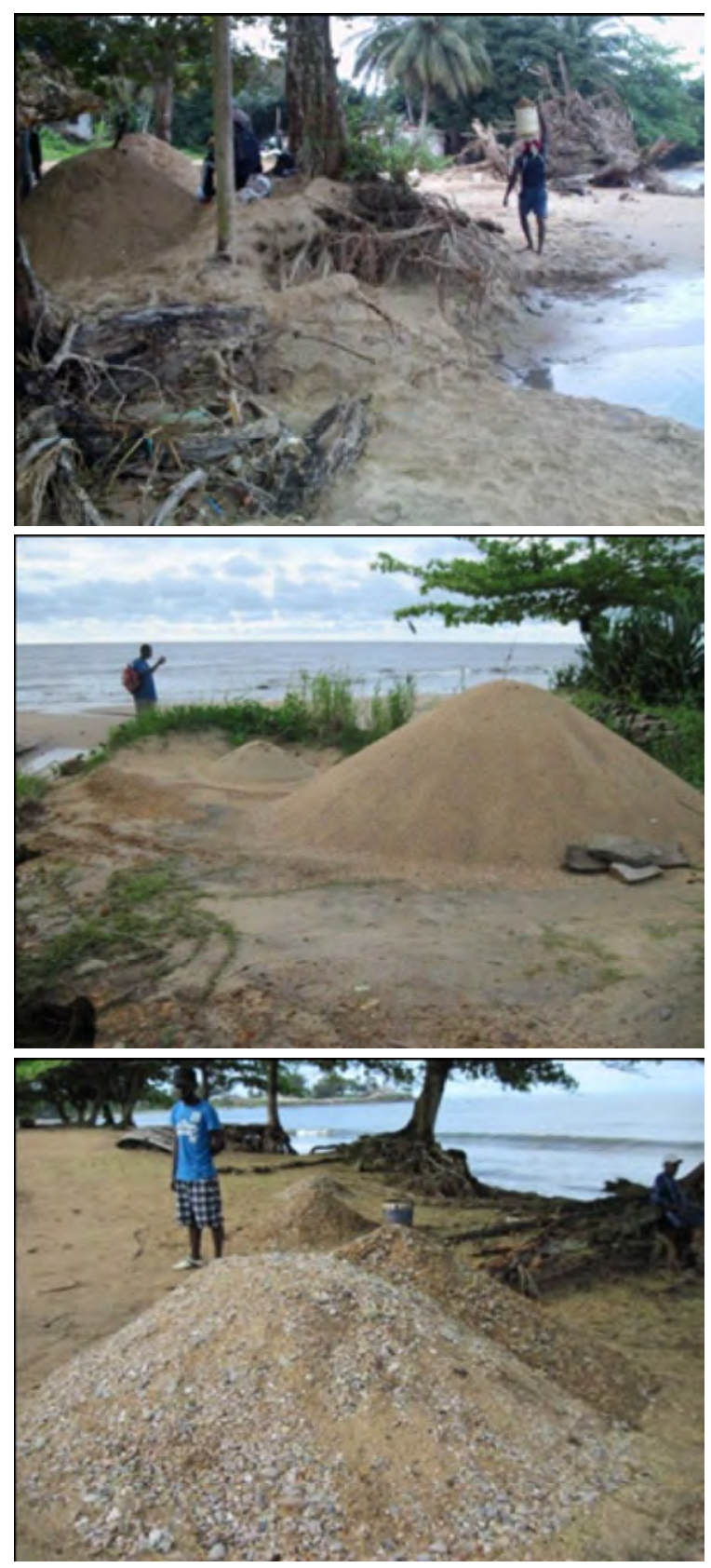

Fig.14 : Photo 13, 14,15 : L'extraction du sable sur les côtes de Kribi.
La figure 14 présente des collines de sables exploitées à des fins de travaux de construction.

\section{a) Non-respect de la réglementation de l'urbanisme}

Le constat fait dans la ville de Kribi est que les constructions ne respectent pas la norme qui stipule que les constructions doivent se faire audelà de $50 \mathrm{~m}$ (100 pas). Des bâtiments ont été observés à quelques mètres de la côte. Ces derniers subissent les mouvements des vagues et par leur disposition, modifient le trait de côte. Ces construction donnent parfois l'illusion que l'homme peut dompter la mer, mais cette dernière disparait à chaque fois que des infrastructures se retrouvent immergées ou presque. D'où l'expression vendeuses «pied dans l'eau » qui apporte une plus-value immobilière à Kribi. (fig.15A et 15B)
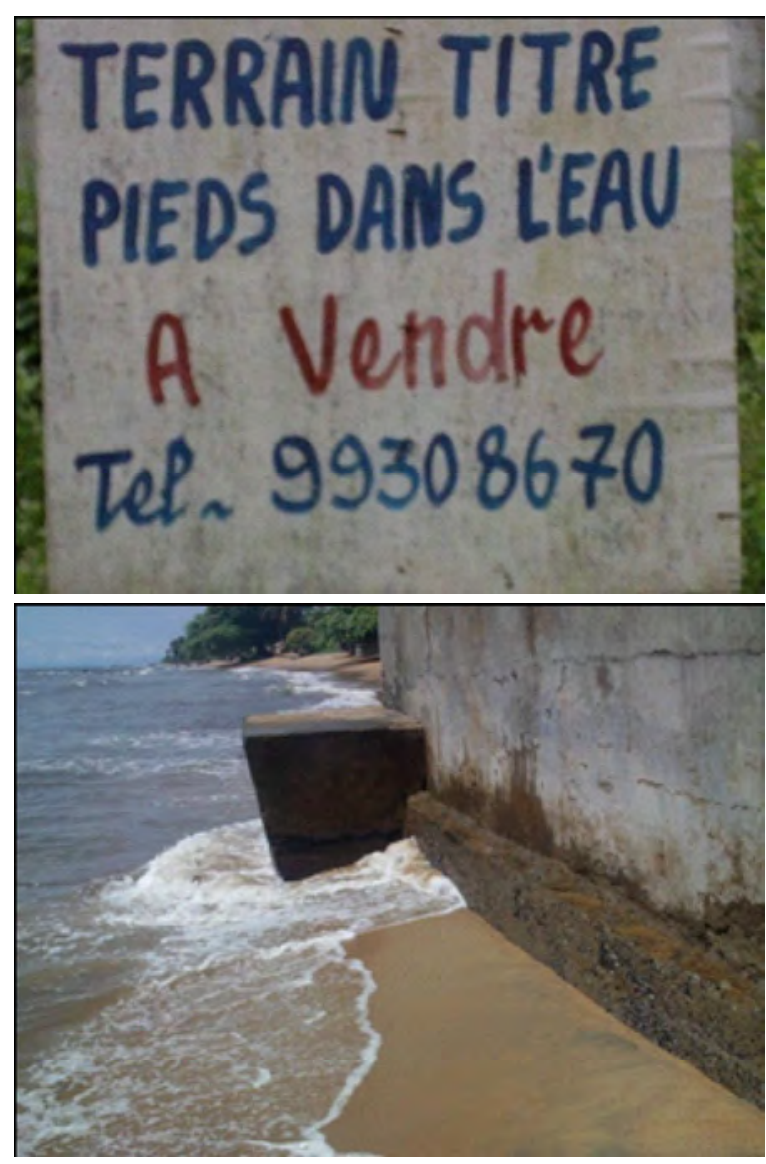

Fig. 15A, 15B: Les constructions en bordure de mer. 
Les figures $15 \mathrm{~A}$ et $15 \mathrm{~B}$ montrent que les individus n'ont pas conscience de la norme qui demande de construire à une distance d'au moins 50 mètres de la côte. Certaines de ces propriétés illégales disposent même des titres fonciers. L'expression «pied dans l'eau » est utilisée comme un slogan et parait fort attrayant. Les bâtiments ainsi construits endiguent l'activité normale des vagues et contribue aussi à modeler le trait de côte. Ces comportements prédisposent donc les infrastructures et les hommes à un certain nombre de risques.

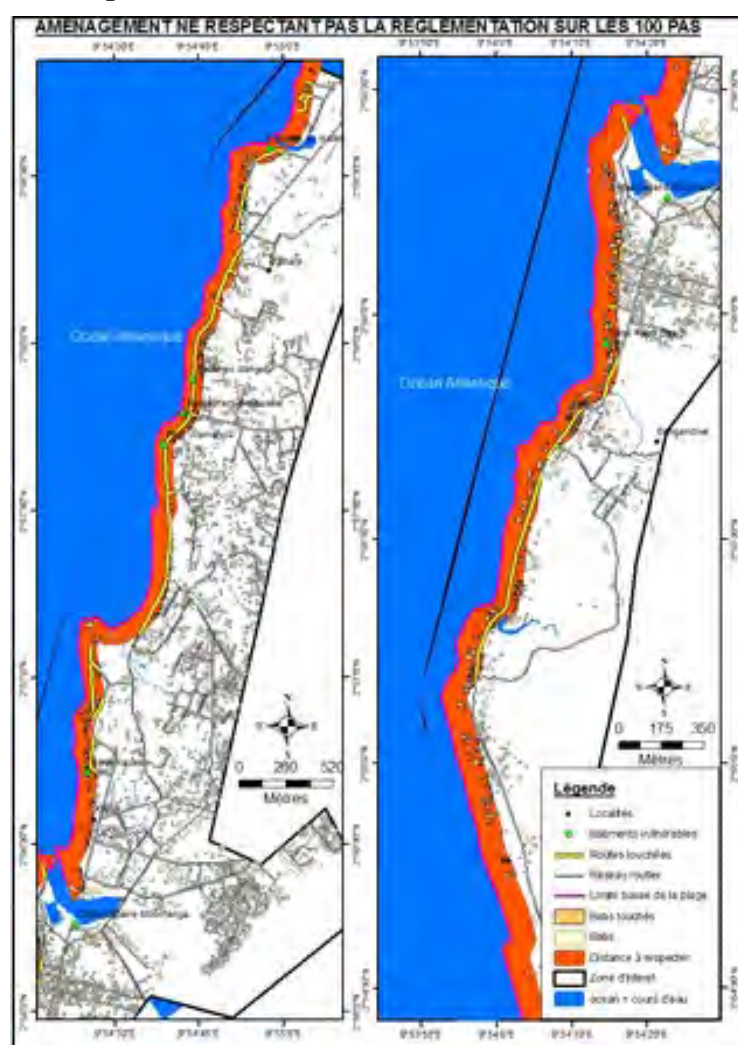

Fig.16 : Aménagements hors normes sur la côte de Kribi

Il ressort de cette figure 16 que de nombreux bâtiments sont situés dans la zone rouge. Ces bâtiments sont pour la plupart des restaurants et infrastructures hôtelières appartenant à des personnalités de la république. Toutes ces infrastructures tombent dans la zone orange qui indique la zone des 100 pas qu'il faut respecter pour toute construction au bord de mer. Mais le constat est que la plupart des bâtiments sur le long de trait de cotes ne respectent pas cette réglementation et contribue à amplifier l'érosion côtière.

\section{CONCLUSIONS}

En définitive, il convient de préciser que la dynamique du trait de côte à Kribi est une réalité dont les répercussions sur les habitats naturels et les hommes sont nombreuses. Cependant, cette dynamique est fille d'un certain nombre de facteurs naturels et anthropiques. Kribi, ville touristique par excellence de nombreux enjeux face à la dynamique du trait de côte. Les études empiriques ont montré que les établissements hôteliers et de restauration construits le long des plages, au sein de la zone interdite de construction, subissent la fureur des vagues qui grignotent progressivement le continent. Ainsi, qu'il s'agisse des routes, des bâtiments et écosystèmes côtiers, rien ne résiste à la force de la dynamique qui gagne de plus en plus du terrain. Mettre en évidence ce risque a nécessité l'utilisation de la télédétection aérospatiale et un travail de terrain minutieux. A l'issu de ce travail les résultats majeurs ont été des cartes de synthèse et d'analyse pouvant permettre aux décideurs de la ville de pouvoir prendre des mesures allant dans le sens de la gestion durable et intégrée de l'écosystème côtier de Kribi qui subit des effets néfastes. Il est à relever que les populations ou encore les opérateurs économiques qui sont les mieux représentés sur la zone rouge ont développé des techniques pour réduire leur vulnérabilité mais ces mesures restent encore provisoires. L'accent devrait être en priorité mis sur la sensibilisation des populations et promoteurs immobiliers sur les enjeux de la bonne gestion de ce trait de côte. Les autorités administratives et locales devraient passer à la phase répressive pour dissuader des futures constructions ou aménagement dans la zone interdite. 


\section{Remerciements}

Les auteurs tiennent à remercier particulièrement le Prof Tchawa Paul pour son encadrement et la relecture du travail. Nos remerciements vont aussi à l'endroit de $\mathrm{Mr}$ Sitio Denis pour sa contribution et son support financier pour la phase de terrain. Notre remerciement spécial à IGCP School de l'UNESCO pour la contribution unique afin de parfaire cet article. Nous sommes reconnaissants à Madame Mokam Simo Aurelie pour ses commentaires et la relecture du travail.

\section{BIBLIOGRAPHIE}

Articles et journaux

Akono, A., Tonyé, E., \& Yossi, R. N. Étude comparative de quatre techniques de multirésolution par ondelettes pour la classification des images radar à synthèse d'ouverture. Télédétection, 2(4), 267-277.

Akono, A., Tonyé, E., Passo, M. M. D., Tankam, N. T., \& Dzepa, C. (2007). Détermination d'une ligne de rivage de grande taille à l'aide de la multi résolution hiérarchique sur une image RSO Determination of large scale shorelines using hierarchical multi resolution on an RSO image. Cybergeo: European Journal of Geography. 110

Chesneau, E. (2004). Propositions pour une cartographie du risque. Le monde des cartes, 181, 55-70.

Ioane, D., Diaconescu, M., Chitea, F., \& Garbacea, G. (2014). Active fault systems and their significance for urban planning in Bucharest, Romania. In Earthquake hazard impact and urban planning (pp. 15-43). Springer, Dordrecht.

Mbuh, M. J., Mayer, R. É., \& Paul, T. (2011). Assessment of the Nature and the Rate of Coastal Erosion on the Mount Cameroon Coastal Landscape, Southwest Region, Cameroon. Journal of Coastal Research, 28(5), 1214-1224.
Morin, S., \& Kuete, M. (1989). Le littoral camerounais: Problèmes morphologiques. Trav. Labo. Géogr. Phys. appliquée. Institut de Géographie, Université de Bordeaux, 3(11), 5-53.

Nfomou, N., Tongwa, A. F., Ubangoh, U. R., Bekoa, A., Metuk, N. J., \& Victor, H. J. (2004). The July 2002 earthquake in the Kribi region: geological context and a preliminary evaluation of seismic risk in southwestern Cameroon. Journal of African Earth Sciences, 40(3-4), 163-172.

Ngaha, P. R. N., \& Belinga, S. M. E. (1987). The diachronism of the basal sandstone, paleoenvironment and the role of the opening of the South Atlantic. Annals of the Faculty of Sciences, Earth Science, Series IV, 1(3-4), 103-119.

Paskoff, R. (2010). Les littoraux: impact des aménagements sur leur évolution. Armand Colin.

Paul Tchawa, Plan d'aménagement et de gestion de la bande côtière Kribi-Campo'o dans la perspective d'un tourisme durable, SNV-Cameroun, 2004. 1-106.

Sikirou Kolawole, A (1997) : Vers une gestion intégrée de la zone côtière du Golfe de la Guinée - Document Cadre. ONUDI ; Les éditions du Flamboyant.

Tiafack, O., Chrétien, N., \& Emmanuel, N. N. (2014). Development Polarisation in Limbe and Kribi (Littoral Cameroon): Growth Challenges, Lessons from Douala and Options. Current Urban Studies, 2(04), 361.

Tonye, Emmanuel., Akono Alain., Nyoungui, A., Nlend, C., \& Rudant, J. (2000). Cartographie de la ligne de rivage par analyse texturale d'images radar Ã synthèse d'ouverture de ERS-1 et de ESAR. Télédétection, 1, 182-204.

Trebossen, H., Rudant, J. P., Fruneau, B., \& Classeau, N. (2000). Contribution of RADAR SAT Imagery for Mapping Coastal Areas: Examples of Sedimentational and Erosional 
Zones in French Guyana, Mauritania. In $S A R$ workshop: CEOS Committee on Earth Observation Satellites (Vol. 450, p. 437).

\section{Rapports autres documents consultes}

Caran, C. S., Woodruff Jr, C. M., \& Thompson, E. J. (1981). Lineament Analysis and Inference of Geologic Structure-Examples from the Balcones/Ouachita Trend of Texas (1).

Dossier de faisabilité - Etude d'impacts environnementaux (EIE) du projet pipeline Tchad-Cameroun, 1996.

Gazel, J., Hourcq, V., \& Nickles, M. (1956). Notice explicatif de la carte géologique a 1/1000 000 du Cameroun. Bull. DMG, Yaoundé, (2).

Hubert, F. N., \& Paul, Tchawa Observation et analyse des paysages littoraux de la région kribienne à partir d'un Système d'Information Environnemental basé sur les Images ERS et optiques. 226-227.

Ministère de l'environnement et de la protection de la nature, (Mai 2011) Mise en œuvre de la gestion intégrée des zones côtières (GIZC) pour la région de Kribi-Campo au Cameroun, 1-221. Nobuyuki H. (1977): Landforms and superficial deposits in the coastal region of Cameroon. In Geomorphological studies in the forest and savanna areas of Cameroon, Edited by Hiroshi Kadomura (An interim Report of the Tropical African Geomorphology Research project. 1975-76).

ONUDI (1982): Pollution de la côte ouest africaine par hydrocarbure, ONUDI.

ONUDI / Cameroun : Profil Côtier du Cameroun.

SUCHEL J. B. les climats du Cameroun. Thèse de doctorat d'état, 4 tomes, Bordeaux, (1988) 88p + atlas.

Zogning A. Et M Kuete., (1985) L'équilibre écologique du littoral camerounais. Données géographiques du problème. Xème Co 